\title{
BOUNDED REGULAR SETS ${ }^{1}$
}

\section{SEYMOUR GINSBURG AND EDWIN H. SPANIER}

Introduction. Earlier [2] we introduced the bounded context free languages (also called "bounded ALGOL-like languages"). (The context free languages are good approximations to the syntactic classes which arise in currently used programming languages.) A most important class of context free languages is the class of regular sets. ${ }^{2}$ Because of this it seems appropriate to consider the class of bounded regular sets. In this note we give two characterizations of these sets. One of these characterizations is related to a characterization of bounded context free languages given in [3]. We also relate certain bounded regular sets to their commutative closures.

1. Two characterizations. Let $\Sigma$ be a finite nonempty set and $\Sigma^{*}$ the free semigroup with identity $\epsilon$ generated by $\Sigma$. We shall deal with subsets of $\Sigma^{*}$. If $A$ is a subset of $\Sigma^{*}$, we use $A^{*}$ to denote the subsemigroup with identity (contained in $\Sigma^{*}$ ) generated by $A$. In case $A$ consists of a single word $w$, we also write $w^{*}$ for this subsemigroup.

A set $X$ is said to be bounded if there exist a finite number of words $w_{1}, \cdots, w_{k}$ such that $X \subseteq w_{1}^{*} \cdots w_{k}{ }^{*}{ }^{3}$

We shall be concerned with bounded regular sets. In this section we present two characterizations of bounded regular sets.

A set $X$ is said to be commutative if $x y=y x$ for all $x, y$ in $X$.

Lemмa 1.1. Let $Z$ be the smallest family of sets which contain all finite sets, all sets $w^{*}$ (w in $\Sigma^{*}$ ), and which is closed with respect to finite union and finite product. Then $Z$ contains every commutative regular set.

Proof. Let $R$ be a commutative regular set. By Lemma 5.2 of [2], there exists a word $w$ such that $R \subseteq w^{*}$. Since $R$ is regular, the set of integers $R^{\prime}=\left\{n \mid w^{n}\right.$ in $\left.R\right\}$ is ultimately periodic. ${ }^{4}$ Therefore

Received by the editors February 4, 1966.

1 The research reported in this paper was sponsored in part by the Air Force Cambridge Reseach Laboratories, Office of Aerospace Research, under Contract AF 19(628)-5166, CRL-Algorithmic Languages Program.

${ }^{2}$ For the definitions of regular set and automaton, as well as their interrelations, see [9].

${ }^{3}$ If $X_{1}, \cdots, X_{k}$ are sets of words, then $X_{1} \cdots X_{k}$, called the product, is the set $\left\{x_{1} \cdots x_{k} \mid\right.$ each $x_{i}$ in $\left.X_{i}\right\}$.

${ }^{4}$ In case $w$ is a letter, this is proved in [6]. For arbitrary $w$, the result follows from the case for letters and the fact that the inverse of a homomorphism maps a regular set to a regular set [1]. 
there exist finite sets $I, J$ of nonnegative integers and a positive in teger $k$ such that

$$
R^{\prime}=I \cup\{j+i k \mid j \text { in } J, i \geqq 0\} .
$$

Let $F=\left\{w^{i} \mid i\right.$ in $\left.I\right\}$. Then $F$ is finite. For each $j$ in $J$ let $R_{j}=w^{j}\left(w^{k}\right)^{*}$. Since $R=F \cup \cup_{j \text { in } J} R_{j}, R$ is in $Z$.

CoROLlary. $Z$ is the smallest family of sets which contains all finite sets, all sets $R^{*}, R$ a commutative regular set, and which is closed with respect to finite union and finite product.

Proof. Let $Z^{\prime}$ be the smallest family of sets which contain all finite sets, all sets $R^{*}, R$ a commutative regular set, and which is closed with respect to finite union and finite product. Clearly $Z \subseteq Z^{\prime}$. To prove the converse, it suffices to show that $Z$ contains all sets $R^{*}$, $R$ a commutative regular set. Let $R$ be a commutative regular set. Then there exists a word $w$ such that $R \subseteq w^{*}$. Since $R^{*} \subseteq\left(w^{*}\right)^{*}=w^{*}$, $R^{*}$ is a commutative regular set. By Lemma 1.1, $Z$ contains $R^{*}$.

We now derive our first characterization result.

THEOREM 1.1. $R$ is a bounded regular set if and only if $R$ is in the family $Z$ of Lemma 1.1.

Proof. Obviously every set in $Z$ is regular. By Theorem 3.1 of [2], every set in $Z$ is also bounded. Thus each set in $Z$ is bounded regular.

To see the converse, let $R$ be a bounded regular set. Then, since $R$ is regular, there exists a finite sequence $X_{1}, \cdots, X_{m}$ of families of sets such that

(1) $X_{1}$ is a finite collection of finite sets.

(2) $R$ is in $X_{m}$.

(3) For $2 \leqq i \leqq m, X_{i}$ is obtained by adjoining to $X_{i-1}$ a set $A_{i}$ which is either the union or the product of two sets in $X_{i-1}$, or is $E^{*}$ for some set $E$ in $X_{i-1}$. For each $i$ let $Y_{i}$ be the collection of bounded sets in $X_{i}$. Then $Y_{1}=X_{1}$. Since $R$ is bounded, $R$ is in $Y_{m}$.

Consider $Y_{i}, 2 \leqq i \leqq m$. It is clear that either $Y_{i}=Y_{i-1}$ or $Y_{i}=Y_{i-1} \cup\left\{A_{i}\right\}$. If $A_{i}$ is not bounded, then $Y_{i}=Y_{i-1}$. If $A_{i}$ is bounded, then either $A_{i}=B_{1} \cup B_{2}$ or $A_{i}=B_{1} B_{2}$ or $A_{i}=B_{3}{ }^{*}$ for $B_{1}, B_{2}$ (or $B_{3}$ ) in $X_{i-1}$. In the first two cases, both $B_{1}$ and $B_{2}$ must be bounded, thus in $Y_{i-1}$. In the last case, since $A_{i}$ is bounded, it follows from Lemma 5.3 of [2] that $B_{3}$ is commutative. In any case, either $Y_{i}=Y_{i-1}$ or $Y_{i}$ is obtained from $Y_{i-1}$ by adjoining a set $A_{i}$ which is either the union or the product of two sets in $Y_{i-1}$ or is $C^{*}$ for some 
commutative regular set $C$ in $Y_{i-1}$. Thus $R$ is in $Z$ by the corollary of Lemma 1.1.

COROLlaRY. $R$ is bounded regular if and only if $R$ is in the smallest family of sets containing all regular subsets of $w^{*}, w$ in $\Sigma^{*}$, and closed with respect to finite union and finite product.

Proof. Let $W$ be the smallest family of sets containing all regular subsets of $w^{*}, w$ in $\Sigma^{*}$, and closed with respect to finite union and finite product. Then $W$ contains $Z$ of Lemma 1.1. Since each set in $W$ is also bounded regular, $W=Z$.

We now derive our second characterization of bounded regular sets.

Lemma 1.2. Let $\Sigma=\left\{a_{1}, \cdots, a_{n}\right\}$. A subset $X$ of $a_{1}{ }^{*} \cdots a_{n}{ }^{*}$ is regular if and only if it is a finite union of sets of the form

(1) $A_{1} \cdots A_{n}$, where each $A_{i}$ is a regular subset of $a_{i}{ }^{*}$.

Proof. If $X$ is such a finite union, it is clearly regular. We prove the converse. Since multiplication is distributive with respect to union, it follows from the previous corollary that each bounded regular set is a finite union of sets of the form

(2) $B_{1} \cdots B_{m}$,

where each $B_{i}$ is a regular subset of $w_{i}^{*}$ for some $w_{i}$ in $\Sigma^{*}$. Thus any regular subset of $a_{1}{ }^{*} \cdots a_{n}{ }^{*}$ is a finite union of sets of the form (2). Obviously each corresponding word $w_{i}$ is either a power of some $a_{j}$ or is a product $a_{i_{1}} \cdots a_{i_{k}}$, with $i_{1} \leqq i_{2} \leqq \cdots \leqq i_{k}$. Thus each set of the form (2) can be written as

(3) $C_{1} \cdots C_{r}$,

where each $C_{i}$ is a regular subset of $a_{f(i)}^{*}$ for some $f(i), 1 \leqq f(i) \leqq n$. For each $i, 1 \leqq i \leqq n$, let

(4) $A_{i}=C_{i 1} \cdots C_{i g(i)}$,

the $C_{i j}$ being just those $C_{k}$ which are subsets of $a_{i}^{*}$. Let $A_{i}=\{\epsilon\}$ if there is no $i$ such that $C_{i} \subseteq a_{i}^{*}$. Then

(5) $A_{1} \cdots A_{n}=C_{1} \cdots C_{r}$, so that $X$ has the prescribed form.

Using the language of [3], in particular, the notion of a linear set, we can reinterpret the lemma as follows.

Corollary. Let $\Sigma=\left\{a_{1}, \cdots, a_{n}\right\}$. Then $R \subseteq a_{1}{ }^{*} \cdots a_{n}{ }^{*}$ is regular if and only if $\left\{(i(1), \cdots, i(n)) \mid a_{1}^{i(1)} \cdots a_{n}{ }^{i(n)}\right.$ in $\left.R\right\}$ is a finite union of linear sets $L_{k}$, each period in each $L_{k}$ having at most one nonzero coordinate. 
Using the lemma we now prove

TheOREM 1.2. A subset $X$ of $w_{1}^{*} \cdots w_{r}^{*}$ is regular if and only if it is a finite union of sets of the form $X_{1} \cdots X_{r}$, where each $X_{i}$ is a regular subset of $w_{i}^{*}$.

Proof. Let $b_{1}, \cdots, b_{r}$ be $r$ distinct symbols not in $\Sigma$. Let $h$ be the homomorphism which maps each $b_{i}$ into $w_{i}$. Since the inverse of a homomorphism maps a regular set to a regular set $[1], h^{-1}(X)$ is regular. Thus $Y=h^{-1}(X) \cap b_{1}{ }^{*} \cdots b_{r}{ }^{*}$ is regular. Clearly

$$
Y=\left\{b_{1}^{i(1)} \cdots b_{r}^{i(r)} \mid w_{1}^{i(1)} \cdots w_{r}^{i(r)} \text { in } X\right\} .
$$

By Lemma 1.2, $Y$ is a finite union of sets of the form $Y_{1} \cdots Y_{r}$, each $Y_{i}$ a regular subset of $b_{i}{ }^{*}$. Then $X=h(Y)$ is a finite union of sets of the form $h\left(Y_{1}\right) \cdots h\left(Y_{r}\right)$, each $h\left(Y_{i}\right)$ a regular subset of $w_{i}^{*}$.

From the corollary to Lemma 1.2 and the fact that

$$
Y=\left\{b_{1}^{i(1)} \cdots b_{r}^{i(r)} \mid w_{1}^{i(1)} \cdots w_{r}^{i(r)} \text { in } X\right\}
$$

is regular we get the following which is related to Theorem 2.1 of [3].

Theorem 1.3. Let $X \subseteq w_{1}^{*} \ldots w_{r}^{*}$ each $w_{i}$ in $\Sigma^{*}$. A necessary and sufficient condition that $X$ be regular is that

$$
\left\{(i(1), \cdots, i(r)) \mid w_{1}^{i(1)} \cdots w_{r}^{i(r)} \text { in } X\right\}
$$

be a finite union of linear sets $L_{k}$, each period in each $L_{k}$ having at most one nonzero coordinate.

2. Commutative closure. We now give a condition for a subset $X$ of $a_{1}{ }^{*} \cdots a_{n}{ }^{*}$, the $a_{i}$ being distinct symbols, to be regular in terms of the "commutative closure" of $X$.

Definition. The commutative closure $c(X)$ of $X \subseteq \Sigma^{*}$ is the set of all words $x_{1} \cdots x_{k}$, each $x_{i}$ in $\Sigma$, such that for some permutation $\tau$ of $\{1, \cdots, k\}$ the word $x_{\tau(1)} \cdots x_{\tau(k)}$ is in $X .(c(X)$ contains $\epsilon$ if and only if $X$ contains $\epsilon$.)

In general, $X$ may be regular without $c(X)$ being regular. For example, let $\Sigma=\{a, b\}$. Then $X=(a b)^{*}$ is regular but $c(X)$ is not regular. For if $c(X)$ were regular, then $c(X) \cap a^{*} b^{*}=\left\{a^{n} b^{n} \mid n \geqq 0\right\}$ would be regular, a contradiction.

Theorem 2.1. Let $\Sigma=\left\{a_{i} \mid 1 \leqq i \leqq n\right\}$ and $X$ a subset of $a_{1}{ }^{*} \cdots a_{n}{ }^{*}$. Then $X$ is regular if and only if $c(X)$ is regular.

Proof. Suppose $c(X)$ is regular. Then $X=a_{1}{ }^{*} \cdots a_{n}{ }^{*} \cap c(X)$. 
Since the intersection of regular sets is regular, $X$ is regular.

Suppose $X$ is regular. By Lemma 1.2, $X$ is a finite union of sets of the form $X_{1} \cdots X_{n}$, where each $X_{i}$ is a regular subset of $a_{i}{ }^{*}$. It thus suffices to prove that if $X$ is of the form $X_{1} \cdots X_{n}$, each $X_{i}$ a regular subset of $a_{i}{ }^{*}$, then $c(X)$ is regular. For each $i$, let $A_{i}=\left(K_{i}, \Sigma, \delta_{i}, s_{i 0}, F_{i}\right)$ be an automaton such that $T\left(A_{i}\right)=X_{i}$. Let $A$ be the automaton $\left(K, \Sigma, \delta, s_{0}, F\right)$, where $K=K_{1} \times \cdots \times K_{n}, s_{0}=\left(s_{10}, \cdots, s_{n 0}\right)$, $F=F_{10} \times \cdots \times F_{n 0}$, and

$$
\delta\left(\left(s_{1}, \cdots, s_{n}\right), a_{i}\right)=\left(s_{1}, \cdots, s_{i-1}, \delta_{i}\left(s_{i}, a_{i}\right), s_{i+1}, \cdots, s_{n}\right) .
$$

It is readily seen that a word $w$ in $\Sigma^{*}$ is in $T(A)$ if and only if, for each $i, a_{i}^{h_{i}(w)}$ is in $T\left(A_{i}\right)$ where $h_{i}(w)$ is the number of occurrences of $a_{i}$ in $w$. Therefore $T(A)=c(X)$, so that $c(X)$ is regular.

Note that Theorem 2.1 does not state that the commutative closure of a bounded regular set is regular. In fact, the example prior to Theorem 2.1 furnishes a bounded regular set whose commutative closure is not regular.

3. Decidability. We now consider the problem of deciding whether a given set is a bounded regular set. It follows from Theorem 5.2 of [2] that it is decidable whether or not a given regular set is bounded. We show that it is decidable of a given semilinear ${ }^{5}$ subset $L$ of $N^{n}$ whether $\tau^{-1}(L)$ is regular (where $\tau$ is the function which maps a word $a_{1}^{i_{1}} \cdots a_{n}^{i_{n}}$ of $a_{1}{ }^{*} \cdots a_{n}{ }^{*}$ into the $n$-tuple $\left(i_{1}, \cdots, i_{n}\right)$ of $\left.N^{n}\right)$. In view of Theorem 2.1, this is equivalent to the condition that $c\left(\tau^{-1}(L)\right)$ is regular.

We first obtain a necessary and sufficient condition on $L$ for $c\left(\tau^{-1}(L)\right)$ to be regular. For this we introduce the following relation on elements of $N^{n}$.

Notation. Let $L$ be a subset of $N^{n}$. For $x, x^{\prime}$ in $N^{n}$, write $x \equiv^{L} x^{\prime}$ if, for all $u$ in $N^{n}$,

$$
x+u \text { is in } L \text { if and only if } x^{\prime}+u \text { is in } L .
$$

Clearly $\equiv^{L}$ is an equivalence relation.

Lemma 3.1. Let $L$ be a subset of $N^{n}$. Then $c\left(\tau^{-1}(L)\right)$ is regular if and only if there are a finite number of equivalence classes generated by the equivalence relation $\equiv L$.

Proof. Let $X=c\left(\tau^{-1}(L)\right)$. Now $X$ is regular if and only if there are a finite number of equivalence classes of $\Sigma^{*}$ generated by the following equivalence relation $\sim^{x}: w \sim^{x} w^{\prime}$ if and only if, for all words

5 The reader is referred to [3] for the notation and concepts used in this section. 
$w^{\prime \prime}$ in $\Sigma^{*}, w w^{\prime \prime}$ is in $X$ if and only if $w^{\prime} w^{\prime \prime}$ is in $X$ [9]. Since $X$ is the commutative closure of $\tau^{-1}(L)$ it follows that if $w$ is any word in $\Sigma^{*}$ and $w^{\prime}$ is an arbitrary permutation of $w$, then $w \sim^{x} w^{\prime}$. Therefore every equivalence class has a representative which is a word in $a_{1}{ }^{*} \cdots a_{n}{ }^{*}$. Thus $X$ is regular if and only if there are a finite number of equivalence classes of $a_{1}{ }^{*} \cdots a_{n}{ }^{*}$ generated by $\sim^{x}$.

To complete the proof we verify that for $w$ and $w^{\prime}$ in $a_{1}{ }^{*} \cdots a_{n}{ }^{*}$, $w \sim^{X} w^{\prime}$ if and only if $\tau(w) \equiv^{L} \tau\left(w^{\prime}\right)$. Let $w$ and $w^{\prime}$ be in $a_{1}{ }^{*} \cdots a_{n}{ }^{*}$. Suppose $w \sim^{x} w^{\prime}$. Let $u$ be an arbitrary element of $N^{n}$ and $y$ in $\tau^{-1}(u)$. Then $\tau(w)+u$ is in $L$ if and only if some permutation of $w y$ is in $\tau^{-1}(L)$, and $\tau\left(w^{\prime}\right)+u$ is in $L$ if and only if some permutation of $w^{\prime} y$ is in $\tau^{-1}(L)$. Therefore $\tau(w)+u$ (or $\tau\left(w^{\prime}\right)+u$ ) is in $L$ if and only if $w y$ (or $w^{\prime} y$ ) is in $X$. Thus $\tau(w) \equiv L \tau\left(w^{\prime}\right)$. Conversely, suppose $\tau(w) \equiv^{L} \tau\left(w^{\prime}\right)$. Then for each element $y$ in $a_{1}{ }^{*} \cdots a_{n}{ }^{*}, w y$ is in $X$ if and only if $w^{\prime} y$ is in $X$. If $z$ is an arbitrary element of $\Sigma^{*}$, then some permutation of $z$ is an element $y$ in ${a_{1}}^{*} \cdots a_{n}{ }^{*}$. Since $X$ is a commutative closure, $w z$ (or $w^{\prime} z$ ) is in $X$ if and only if $w y$ (or $w^{\prime} y$ ) is in $X$. Therefore $w \sim^{x} w^{\prime}$ and the proof is complete.

COROllary. It is decidable to determine for an arbitrary semilinear set $L$ in $N^{n}$ whether $c\left(\tau^{-1}(L)\right)$ is regular.

Proof. We use a method found in [5]. By Theorem 1.3 of [3], a Presburger formula $P\left(x_{1}, \cdots, x_{n}\right)$ over the nonnegative integers can be effectively found such that $L=\left\{\left(x_{1}, \cdots, x_{n}\right)\right.$ in $N^{n} \mid$ $P\left(x_{1}, \cdots, x_{n}\right)$ is true $\}$. Let $Q$ be the following Presburger sentence:

$$
\begin{gathered}
(\exists k)\left(x_{1}\right) \cdots\left(x_{n}\right)\left(\exists x_{1}^{\prime}\right) \cdots\left(\exists x_{n}^{\prime}\right) \\
{\left[\bigwedge _ { i = 1 } ^ { n } ( x _ { i } ^ { \prime } \leqq k ) \wedge ( y _ { 1 } ) \cdots ( y _ { n } ) \left(P\left(x_{1}+y_{1}, \cdots, x_{n}+y_{n}\right)\right.\right.} \\
\left.\left.\equiv P\left(x_{1}^{\prime}+y_{1}, \cdots, x_{n}^{\prime}+y_{n}\right)\right)\right] .
\end{gathered}
$$

Then $Q$ is true if and only if there are a finite number of equivalence classes of $N^{n}$ generated by $\equiv L$. By Lemma 3.1, this is equivalent to the condition that $c\left(\tau^{-1}(L)\right)$ is regular. Since the truth of every Presburger sentence is decidable [4], the corollary holds.

We now obtain the following result also due to [5] and [8].

THEOREM 3.1. It is decidable to determine for an arbitrary regular set $R$ whether $c(R)$ is regular. 
Proof. Let $R \subseteq \Sigma^{*}$ be regular and $\psi$ the Parikh mapping of $\Sigma^{*}$ into $R$. Then $L=\psi(R)$ is semilinear [7]. Since $c(R)=c\left(\tau^{-1}(L)\right)$, the result follows from the preceding corollary.

\section{REFERENCES}

1. S. Ginsburg and G. F. Rose, Operations which preserve definability in languages, J. Assoc. Comput. Mach. 10 (1963), 175-195.

2. S. Ginsburg and E. H. Spanier, Bounded ALGOL-like languages, Trans. Amer. Math. Soc. 113 (1964), 333-368.

3. - Semigroups, Presburger formulas, and languages, Pacific J. Math. 16 (1966), 285-296.

4. D. Hilbert and P. Bernays, Grundlagen der Mathematik, Edward Brothers Inc., Ann Arbor, Mich., 1944.

5. R. Laing and J. B. Wright, Commutative machines, Tech. Rep., Univ. of Michigan, Ann Arbor, Mich., December 1962.

6. J. Myhill, Finite automata and the representation of events, WADC Tech. Rep. 57-624, 1957, pp. 112-137.

7. R. J. Parikh, Language-generating devices, Quarterly Progress Rep. No. 60, Research Laboratory of Electronics, January 1961, pp. 199-212, Massachusetts Institute of Technology, Cambridge, Mass.

8. V. N. Redko, On the commutative closure of events, Dopovidi Akad. Nauk Ukrain. RSR (1963), 1156-1159.

9. M. O. Rabin and D. Scott, Finite automata and their decision problems, IBM J. Res. Develop. 3 (1959), 114-125.

System Development Corporation and

UNIVERSiTy OF CALIFORNIA AT BERKELEY 